\title{
Barrismo popular entre la seguridad, comodidad y convivencia: un análisis comparado sobre el diseño de la política del fútbol en Cali y Medellín
}

Popular Cheering Between Security, Comfort and Coexistence: A Comparative Analysis on the Design of Soccer Politics in Cali and Medellín

Barrismo popular entre segurança, conforto e convivência: uma análise comparativa sobre o desenho da política do futebol em Cali e Medellín

\author{
Juan Manuel Restrepo Caicedo \\ Universidad del Cauca (Cauca-Colombia) \\ juanma09restrepo@gmail.com
}

DOI: https://doi.org/10.32719/25506641.2019.5.2

Fecha de presentación: 19 de diciembre de 2018 • Fecha de aceptación: 17 de abril de 2019 Artículo de investigación

Licencia Creative Commons

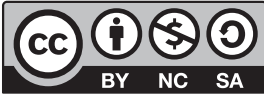




\section{Resumen}

Este artículo analiza cómo la tenue participación de actores no estatales -como las barras populares-, dentro del "Plan Decenal de Seguridad Comodidad y Convivencia en el fútbol (2014-2024)", afecta su proceso de implementación. A partir de la perspectiva de teórica anidada de Howlett y el debate de la gobernanza, se evidencia la fragilidad de la política en cuanto a su instrumentación (específicamente en términos de recursos y organización) y la ausencia de legitimidad y eficacia por la falta de inclusión, y concluye que el desarrollo de canales participativos de carácter interactivo coadyuva a la efectividad de la política. Se realiza un análisis comparativo entre Cali y Medellín.

Palabras clave: Barras populares, fútbol, políticas públicas, violencia.

JEL: I38 Política pública; provisión y efectos de los programas de bienestar; K42 Conducta ilegal y aplicación de la ley.

\section{Abstract}

This article analyzes how the tenuous participation of non-state actors -fan groups (cheering) - within the "Ten-Year Plan for Safety, Comfort and Coexistence in Soccer (20142024)", affects its implementation process. Taking into account the perspective of nested theory developed by Howlett and the debate on governance, the article demonstrates policy fragility regarding to its implementation (specifically in resources and organization terms) and the legitimacy and effectiveness absence due to the lack of inclusion; concluding that the development of interactive participatory channels contributes to policy effectiveness. Methodologically, it does a comparative analysis between Cali and Medellin cases.

Keywords: Popular fan group, soccer, public policies, violence, cheering.

JEL: I38 Public policy, provision and effects of welfare programs; K42 Illegal conduct and law enforcement.

\section{Resumo}

Este artigo analisa como a tênue participação de atores não estatais - bares populares - no desenho do "Plano Decenal de Segurança, Conforto e Convivência no Futebol (20142024)", afeta seu processo de implementação. Do ponto de vista da teoria aninhada desenvolvida por Howlett e do debate sobre governança, o artigo demonstra a fragilidade da política em termos de sua implementação (especificamente em termos de recursos e organização) e a ausência de legitimidade e efetividade devido à falta de inclusão, concluindo que o desenvolvimento de canais participativos interativos contribui para a eficácia da política. Metodologicamente este trabalho parte do análise comparativa entre Cali e Medellín.

Palavras-chave: Bares populares, futebol, políticas públicas, violência.

JEL: I38 Políticas públicas; provisão e efeitos de programas de bem-estar; K42 Conduta ilegal e aplicação da lei. 


\title{
Introducción
}

\begin{abstract}
raíz del proceso de espectacularización facilitado por la globalización y los medios de comunicación, el fútbol como deporte profesional logró transformarse en una de las industrias más rentables de la actualidad. Pese a la rentabilidad de su negocio, el fútbol "apunta precisamente a una experiencia asociativa y al vívido sentido de comunidad que proporciona" (Critchley 2018, 19). Su importancia dentro de la cultura popular y los lazos de afinidad alrededor de la pasión que despierta permitieron la consolidación de espacios de integración, sentidos de pertenencia y la construcción de un sistema identitario agrupado en movimientos sociales denominados barras populares.

"La construcción de identidades a través del fútbol, arraigado a un proceso competitivo entre dos equipos, deja de caracterizar una simple contienda deportiva para transformarse en una lucha que trasciende lo simbólico" (Restrepo 2018, 4). Estas formas de asociación popular alrededor del fútbol, reflejan todo un sistema cultural en el que las barras populares se ven a sí mismas como el baluarte del equipo al cual representan. En consecuencia:
\end{abstract}

Factores como la construcción de identidades, sentidos de poder y procesos de territorialización que están intrínsecamente inmersos dentro de la lógica del funcionamiento de estas barras (sin olvidar los contextos de marginalidad de los que muchas veces proceden sus miembros), son la base principal para el entendimiento de un fenómeno creciente de violencia en el fútbol (Restrepo 2018, 6).

La violencia en el fútbol no solo manifiesta impactos negativos en términos de seguridad y percepción de la misma cuando algunos miembros de las barras desatan peleas o atentan contra la infraestructura privada y pública, sino que también sus efectos se perciben en lo económico, tanto para los 
clubes deportivos como para el comercio formal e informal aledaño a los estadios.

Debido a la regularidad con que este fenómeno se presenta, la respuesta estatal se ha centrado en una actuación coercitiva y restrictiva, recurriendo a medidas como: imposición de rejas en las tribunas, acceso limitado a los escenarios deportivos, sanciones económicas, sistemas de vigilancia y aumento de seguridad pública y privada (Restrepo 2018). Estas medidas, "si bien sirven para apaciguar los brotes de violencia, solo atacan los hechos coyunturales más no resuelven los problemas estructurales" (Restrepo 2018, 7).

Por lo anterior, en Colombia, el "Plan de Seguridad, Comodidad y Convivencia en el Fútbol (2014-2024)" surge como una iniciativa estatal que tiene por objetivo fomentar la cultura de no violencia y ver en el fútbol una salida para la reconstrucción del tejido social (Restrepo 2018). Sin embargo, la efectividad de esta política se ha visto condicionada debido a la pobre interacción entre los distintos actores estatales y no estatales (especialmente con las barras populares), así como la falta de voluntad política para poder implementarse.

Con base en este antecedente, el objetivo de este estudio radica en identificar cómo la falta de coordinación entre diversos actores estatales nacionales con las autoridades locales y una tenue interacción con los actores no estatales (barristas), al implementar la política, deriva en un proceso ineficaz de acción de la misma. Para ello se tomaron como referente los casos de las ciudades de Cali y Medellín.

Esta investigación, como hoja de ruta teórica, partió del modelo anidado multinivel de elección de instrumentos de política y diseño de políticas desarrollado por Howlett (2009). A través de dicha propuesta teórica, es posible observar y analizar los diversos componentes de una política y cómo estos se articulan para alcanzar grados de coherencia y congruencia entre las metas generales, los instrumentos utilizados para alcanzarlas, y su posterior calibración, a medida que la política vaya experimentando cambios. Consecutivamente, se contempla el debate de la gobernanza como un elemento del sistema de administración pública contemporánea que pone de manifiesto la participación de actores no estatales como mecanismo eficaz para el diseñar políticas públicas. 


\section{Metodología}

En cuanto a la metodología, se plantea realizar un análisis comparado, tomando como casos las ciudades de Cali y Medellín a través del sistema más similares propuesto por Pzerworski y Teune (1982), el cual consiste en observar casos que presenten similitudes (políticas, sociales, demográficas, económicas, culturales), pero que varíen en algunos aspectos importantes.

Las técnicas de recolección de datos para este estudio principalmente se basa en fuentes de información primaria y secundaria, recurriendo a entrevistas en profundidad con funcionarios públicos (específicamente las secretarías encargadas del tema de la seguridad), miembros de la policía nacional y líderes de las barras populares. Consecutivamente, se recurrió a revisar la prensa local, los actos administrativos y la bibliografía explícita del fenómeno, con la finalidad de que la información adquirida se pueda validar por medio de su triangulación.

\section{Revisión de literatura}

\section{Coherencia e instrumentación como problema de diseño de política}

El desarrollo de herramientas como políticas públicas es cada vez más relevante para la atención de las necesidades sociales. Sin embargo, la complejidad que conlleva el desarrollo de dichas herramientas implica prestar atención a la elaboración de análisis que contribuyan a identificar los resultados o avances de la aplicación de las mismas. A partir de dicha perspectiva, Howlett (2009) presenta una propuesta de análisis basada en concebir una política como un diseño integral que va desde el proceso de formulación hasta la implementación, considerando la evaluación como un eje implícito en cada fase que compone el diseño. Dicho análisis fundamentado en un diseño integral, reconoce la importancia de identificar la coherencia entre los objetivos de una política con respecto a los medios o instrumentos que se utilicen para alcanzarlos. Con base en lo expuesto, Howlett desarrolla un modelo de 
análisis de políticas de acuerdo a la coherencia y consistencia entre objetivos y medios desde una perspectiva anidada en tres niveles:

1. El nivel macro implica la determinación de los objetivos abstractos de la política y las preferencias generales de implementación, resaltando la importancia de los tipos de gobernanza (autogobierno, jerárquica y cogobernanza) los cuales "involucran diferentes preferencias para los tipos generales de instrumentos de política que se espera alcancen los objetivos generales" (Howlett 2009, 76).

2. El nivel meso se refiere a la selección de los medios o instrumentos entendidos como: "los recursos que los gobiernos tienen a su disposición para alcanzar los objetivos de la política" (Howlett 2009, 81). Los instrumentos o recursos que los distintos gobiernos construyen y poseen se agrupan de la siguiente manera:

Nodalidad: esta categoría hace referencia a la disponibilidad y uso que un gobierno tiene sobre instrumentos de información (campañas de información púbica, censos, encuestas, conformación de comisiones para realizar investigaciones acerca de un problema determinado, etcétera).

Autoridad: instrumentos basados en la autoridad del gobierno. Generalmente implican regulaciones dirigidas a grupos específicos que en caso de un cumplimiento conduce a sanciones (reglas, leyes, normas, decretos).

Tesoro: dicha categoría obedece a la capacidad fiscal del Estado y la experticia del gobierno en recaudar y distribuir fondos públicos (subsidios, impuestos, tasas, incentivos tributarios, préstamos).

Organización: estos instrumentos se basan en la capacidad del gobierno en ofrecer directa o indirectamente bienes o servicios destinados al cumplimiento de objetivos (empresas públicas, servicios en educación, salud, etcétera.) (Howlett 2009, 82).

3. El nivel micro se enfoca en el seguimiento de los objetivos a través de la implementación de instrumentos, $\mathrm{y}$, en caso de su mal funcionamiento, la posibilidad de calibrarlos o modificarlos. Este nivel "implica evaluar diferentes niveles de intensidad administrativa, grados de precisión de focalización, riesgo político y legitimidad, y actividad estatal" (Howlett 2009, 83) para la distinción final del diseño de políticas.

En resumen, el diseño de políticas, entendido como proceso de construcción integral, en el que intervienen diversos actores políticos cuyas expectativas radican en obtener resultados positivos (objetivos) a través de un tipo 
ideal de formulación e implementación (medios), se estructura con base en un modelo anidado (macro, meso, micro) de toma de decisiones (Howlett 2009), explicada en la siguiente matriz:

Tabla 1

Matiz de análisis de diseño de políticas con base en el modelo anidado multinivel

\begin{tabular}{|c|c|c|c|}
\hline \multirow{2}{*}{$\begin{array}{c}\text { Componentes } \\
\text { de política }\end{array}$} & \multicolumn{3}{|c|}{ Niveles de decisión política } \\
\hline & Macro & Meso & Micro \\
\hline Objetivos & $\begin{array}{l}\text { Metas generales } \\
\text { Enunciados generales sobre } \\
\text { los objetivos y ambiciones } \\
\text { del gobierno en un área de } \\
\text { política. }\end{array}$ & $\begin{array}{l}\text { Objetivos } \\
\text { operacionales } \\
\text { Áreas específicas que se } \\
\text { espera que las políticas } \\
\text { aborden para lograr los } \\
\text { fines de la política. }\end{array}$ & $\begin{array}{l}\text { Ajustes específicos } \\
\text { Requisitos específi- } \\
\text { cos, necesarios para } \\
\text { alcanzar los objetivos } \\
\text { de la política en el } \\
\text { territorio. } \\
\end{array}$ \\
\hline Medios & $\begin{array}{l}\text { Preferencias de } \\
\text { implementación } \\
\text { Las preferencias de largo } \\
\text { plazo que tiene el gobierno } \\
\text { en términos de los tipos de } \\
\text { dispositivos organizacionales } \\
\text { que se utilizarán para abordar } \\
\text { los objetivos de las políticas. }\end{array}$ & $\begin{array}{l}\text { Instrumentos } \\
\text { específicos } \\
\text { Los tipos específicos de } \\
\text { instrumentos de gobier- } \\
\text { no que se utilizarán para } \\
\text { abordar los objetivos a } \\
\text { nivel de programas. }\end{array}$ & $\begin{array}{l}\text { Calibraciones del } \\
\text { instrumento } \\
\text { Configuración espe- } \\
\text { cífica de las herra- } \\
\text { mientas necesarias } \\
\text { para alcanzar los } \\
\text { objetivos de política. }\end{array}$ \\
\hline
\end{tabular}

Fuente: adaptado de Howlett (2009).

\section{Gobernanza como enfoque de participación horizontal}

La gobernanza, entendida como un proceso de interacción entre el Estado y los actores no estatales de la sociedad y del mercado en los procesos de acción pública (Aguilar 2006), permite la construcción de un entendimiento integral de los fenómenos sociales; combinando la experiencia empírica con la profesional, para contribuir a la reducción de la incertidumbre al buscar alternativas frente a los problemas sociales, aumentando la legitimidad y tratando de reducir la conflictividad política que de la implementación de medidas se deriva (Córdova 2018). 
Como consecuencia de la volatilidad que encierran los fenómenos sociales, Cerrillo (2005) concibe que para avanzar hacia un proceso de trabajo mancomunado, teniendo en cuenta los intereses que la diversidad de actores persigue, es importante llegar a un estado de consenso en que "los actores asuman su rol correspondiente, se les garanticen las condiciones necesarias y suficientes para poder tomar las decisiones que les correspondan, y se puedan tomar decisiones finales" (Cerrillo 2005, 19). De esta manera, al diseñar una política, esta se pueda ajustar específicamente a las necesidades del fenómeno social que se desea intervenir.

Por lo anterior, la forma de transitar de un modelo de toma de decisiones "unidireccional hacia un modelo bidireccional en el que se tiene en consideración, aspectos, problemas y oportunidades tanto del sistema de gobierno como del sistema a gobernar" (Sánchez 2012, 249), implica tener en cuenta la forma en que el modelo de cogobernanza ${ }^{1}$ es aplicado, ya que los espacios de participación no siempre son los mismos para todos los actores. Al respecto, Santillán (2004) profundiza en el concepto de participación definiéndola en tres tipos:

1. Participación interactiva: participación en conjunto que conduce a planes de acción y a la formación de organizaciones locales nuevas o al fortalecimiento de las ya existentes.

2. Participación consultiva: participación remitida únicamente a la consulta, pero son profesionales externos quienes definen cuáles son los problemas de la comunidad y cuáles son las soluciones.

3. Participación pasiva: participación de carácter informativa en cuya situación las autoridades no toman en cuenta la opinión de la gente (Santillán 2004).

1. Hace referencia a las formas organizadas de interacción de forma horizontal que se rigen bajo principios de coordinación, cooperación y colaboración, es decir, las partes de la sociedad unen esfuerzos con un objetivo común en mente, y con la participación de su identidad y autonomía a este proceso (Sánchez 2012). 


\section{Una nueva forma de vivir el fútbol: surgimiento del barrismo en Cali y Medellín}

El fenómeno de las barras en Colombia, tal y como lo aseveran Pardey, Galeano y Blanco $(2001,25)$, “no surgió por generación espontánea, como muchos suponen, sino que es el resultado de un largo y atropellado proceso que se inició en la década de los noventa con la conformación de barras de corte tradicional". Dicha década sería testigo de la transición de un sector de espectadores inconformes con el sedentarismo, hacia unos espectadores dinámicos comprometidos con una nueva forma de vivir el fútbol, "siendo el punto de partida para la consolidación de un fenómeno cultural de masas, que generaría afinidades, pero también rivalidades" (Restrepo 2018, 75).

"Las barras actualmente cuentan con un estimado de unos 500 a 900 jóvenes organizados, quienes asisten permanentemente, viajan a todas las ciudades del país donde se realicen los partidos de fútbol y algunas veces al exterior" (Rojas 2013, 39). Ese número puede subir dependiendo de cómo el equipo se esté comportando en el campeonato o cada vez que se avecina un clásico; en esas situaciones, "el estadio puede contar con más o menos unos 3.000 a 5.000 jóvenes asistentes a la tribuna" (Rojas 2013, 39).

En el municipio de Cali, esa creciente masa de espectadores -dinámicos denominados barristas- encontraría en los equipos de la ciudad (América de Cali y Deportivo Cali) un escenario predilecto para el desarrollo de una identidad a partir de la pasión por el fútbol. Dicha pasión, manifestada a través de actividades positivas que incluye la música y el arte, evidencia al mismo tiempo un lado negativo expresado en los hechos de violencia y vandalismo.

Debido a los costos de la boletería, junto al ejemplo de las barras en Argentina, las cuales se ubican siempre en las tribunas populares, el nuevo grupo de jóvenes aficionados conformarían por un lado la barra denominada Barón Rojo Sur (BRS), adscrita al equipo América de Cali, y, por el otro, se conformaría el Frente Radical Verde (FRV), seguidora del equipo Deportivo Cali.

Conforme la cultura del barrismo se hacía más popular, aumentaba el número de aficionados dentro de las barras de la ciudad, dificultando la tarea de organizarse. Como consecuencia de ello, las barras se tuvieron que fraccionar en varios subgrupos con sus líderes respectivos; por un lado se 
denominaron "bloques" para BRS, y, por el otro, "legiones" si se habla de FRV distribuidos por las comunas de la ciudad. "Al conformarse los bloques y las legiones, cada uno de estos adquiría un lugar y un espacio dentro de la tribuna, representándose simbólicamente por medio de "trapos" o banderas para ser distinguidos dentro de la barra en general" (Restrepo 2018, 76).

Al igual que en Cali, el caso de la conformación del barrismo en Medellín surgió como un nuevo espacio para que los jóvenes pudieran relacionarse, forjar lazos de amistad y desarrollar una identidad. Las barras más representativas de la ciudad son Los Del Sur (LDS), fiel seguidora del equipo Atlético Nacional y la Rexixtenxia Norte (RXN), perteneciente Deportivo Independiente Medellín, se ven asociados alrededor de "combos"2 y "parches"'. La barra LDS, sin contar las filiales, ${ }^{4}$ está compuesta de alrededor de 85 combos distribuidos entre las comunas que componen la ciudad, "tiene una forma organizativa que se compone de 40 personas que, entre líderes tradicionales de la barra y líderes de los diferentes combos, dirigen la barra. A este grupo se le conoce al interior de la barra como Comité Central" (Domínguez 2009, 4). Por otro lado, la RXN compuesta de 70 parches, funciona de manera jerárquica con los principales líderes de cada parche, los cuales forman grupos de trabajo alrededor de proyectos sociales, productivos, logísticos y culturales.

\section{Plan Decenal de Seguridad, Comodidad y Convivencia: objetivos e instrumentos}

Según el Centro Nacional de Consultoría, para el $94 \%$ de la sociedad colombiana, el fútbol simboliza una actividad de suma importancia dentro de la cultura popular del país (CO Ministerio del Interior 2014). "Frente a las razones por las cuales la gente considera el fútbol importante, un $61 \%$ señaló que contribuye a alejar a los jóvenes de la violencia; un $26 \%$ que le brinda

2. Los combos son los grupos locales pertenecientes a LDS de la ciudad de Medellín (Domínguez 2009, 4).

3. Se denomina parches a los grupos locales de la ciudad de Medellín adscritos a la Rexixtenxia Norte.

4. Las filiales son los grupos regionales ubicados a lo largo y ancho del territorio nacional (Domínguez 2009, 4). 
oportunidades a los jóvenes; un 24\% que recrea a la gente; y un 22\% que une al país" (CO Ministerio del Interior 2014, 48). Bajo esa premisa, el gobierno nacional, junto con diversos actores no estatales (dirigentes deportivos, ONG, barristas, entre otras), se planteó la necesidad de ver al fútbol como una herramienta que sirva para la construcción de paz. En esa medida, el "Plan Decenal de Seguridad, Comodidad y Convivencia en el Fútbol 20142024" (PDSCCF) surge con el objetivo de hacer del fútbol y la pasión que lo envuelve un espacio cohesionador para alentar la cultura ciudadana de la no violencia (CO Ministerio del Interior 2014).

Para el cumplimiento de dicho objetivo, esta política parte de un marco normativo iniciado a partir de la Ley 1270 (CO 2009), la cual dio paso a la creación de la Comisión Nacional de Seguridad, Comodidad y Convivencia en el Fútbol (CNSCCF), organismo encargado de promulgar que "cada municipio o distrito pueda constituir una Comisión Local de Seguridad, Comodidad y Convivencia en el Fútbol (CLSCCF) en cabeza de la alcaldía, cuyas funciones y operaciones estarán fijadas por la Comisión Nacional" (CO Ministerio del Interior 2014, 25). Las distintas CLSCCF fungen como apoyo para "la implementación de políticas, planes y programas, así como en la ejecución de estrategias dirigidas a mantener la seguridad, comodidad y convivencia en la organización y práctica de este espectáculo deportivo" (CO Ministerio del Interior 2014, 8).

Por otro lado, la política proyecta el fortalecimiento del componente de seguridad a través de la labor policial. En cuanto a la consecución de este objetivo, la política se propone modernizar y adoptar sistemas tecnológicos que mejoren la capacidad de respuesta policial en caso de inseguridad o alteración de orden público $\mathrm{y}$, a su vez, pretende la conformación de centros de coordinación y gestión de información en torno a la seguridad y prevención de la violencia.

De la misma forma en que se pretende la modernización del aparato policial, la política contempla la necesidad de modernizar los escenarios deportivos. Para ello, y con base en los estándares de seguridad recomendados por la FIFA, se estableció la instalación de dispositivos de vigilancia, remodelación de los estadios con estándares antisísmicos e individualización de graderías, sistemas biométricos en las entradas y señalización clara de salidas de emergencia (Restrepo 2018). 
Por su parte, esta política contempla el desarrollo de instancias para la atención de los aficionados mediante el establecimiento de la Defensoría del Aficionado. Según la Resolución 1454 de 2014 (CO 2014), dicha instancia de atención adscrita a la Defensoría del Pueblo se planteó como la posibilidad que tienen todas las personas que asisten al estadio de manifestar cualquier tipo de abuso, reclamo o queja y ser tramitada o atendida por un servidor público que estará de servicio cada vez que se dé un partido. Además, tiene a su cargo el desarrollo y posterior divulgación del manual del aficionado, en el cual se establecen los derechos y deberes del hincha (Restrepo 2018).

De igual manera, la política pretende impulsar la investigación social respecto del fútbol y fortalecer el rol de los medios de comunicación en su papel de ofrecer una información adecuada y responsable y no presentar sesgos al tratar temas asociados a la violencia en el fútbol. Asimismo, esta política proyecta estimular el desarrollo social y comunitario por medio de uso recreativo del fútbol para el fomento de valores en entornos escolares y barrios marginales (Restrepo 2018).

Para finalizar, la política en relación con el cumplimiento del objetivo de generar una cultura no violenta apunta hacia la consolidación del "barrismo social". El barrismo social, diseñado y desarrollado por las mismas barras populares, es una iniciativa que ve en la cultura del barrismo una oportunidad para cambiar el flagelo de la violencia y la marginación socioeconómica a través de la música, el arte y los emprendimientos productivos. Con la disposición de las barras de querer aprovechar su cultura para alejar a los jóvenes de la violencia, la política contempla apoyar el desarrollo de liderazgos, proyectos culturales y económicos que se impulsen desde las barras, trabajos mancomunados con las instituciones estatales, fortalecimiento de las organizaciones barristas, el cumplimiento de derechos y deberes y la resignificación cultural y social del barrismo (Restrepo 2018).

En consecuencia, y siguiendo la estructura analítica diseñada por Howlett, el nivel macro correspondiente a los objetivos y lineamientos de la política, el PDSCCF plantea, como objetivo general,

hacer del fútbol profesional y recreativo un deporte integrador, cohesionador y transformador de la comunidad, que se desarrolle de manera pacífica, segura y en convivencia; contribuya a fomentar el desarrollo social y comunitario, promueva la profesionalización 
y el alto desempeño de los jugadores y sea una herramienta de construcción de ciudadanía para la paz y la democracia (CO Ministerio del Interior 2014, 77).

Para el cumplimiento del mencionado objetivo, la política se planteó el fortalecimiento de tres componentes: 1. Seguridad: mejoramiento de la capacidad técnica y tecnológica de vigilancia; 2. Comodidad: adecuación de escenarios deportivos; y 3. Convivencia: barrismo social.

Con respecto al nivel meso, alusivo al diseño y elección de instrumentos, Howlett propone un esquema de clasificación de los mismos, como una forma de separar las herramientas que dispone una política para su ejecución y poder identificar los puntos débiles de la política en caso de necesitar una calibración (nivel micro). Por consiguiente, el PDSCCF presenta la siguiente taxonomía (ver tabla 2).

Pese a que el diseño del PDSCCF - al menos en su fase de formulación- contó con la participación de diversos actores tanto estatales como no estatales, al implementarse, ha experimentado diversas dificultades que están alejando a la política del cumplimiento de sus objetivos. Partiendo de McConnell (2015), una política, por más que sea exitosa en algunos aspectos mínimos, si no logra fundamentalmente los objetivos que los diseñadores se propusieron alcanzar, el soporte de la misma es prácticamente insostenible. Por consiguiente, a pesar del avance de la política en ciertos aspectos como el de la seguridad, con respeto a la adecuación de tecnología de vigilancia y el desarrollo de concejos locales de seguridad, se observa que la violencia es un factor persistente, condicionando a la política hacia el fracaso.

Con la mitad del período transcurrido de su implementación, la política se ha empeñado en fortalecer el tema de la comodidad y seguridad (remitida simplemente a la protección del espectáculo deportivo), más que el componente de convivencia. La instalación de sistemas biométricos, cámaras de seguridad y procesos de carnetización solo ha sido posible en las principales ciudades del país (Barranquilla, Bogotá, Cali y Medellín) por los elevados costos que esto implica. La política (que es nacional) se está sustentando económicamente en lo que puedan hacer los municipios y no en el gobierno central. Además, el fútbol como negocio privado correspondiente a los clubes deportivos y la entidad que los rige, DIMAYOR, es la que en primera instancia debe procurar ofrecer un servicio adecuado, sin embargo, "no aportan recursos para el apoyo de la política, ya sea por problemas económicos o 


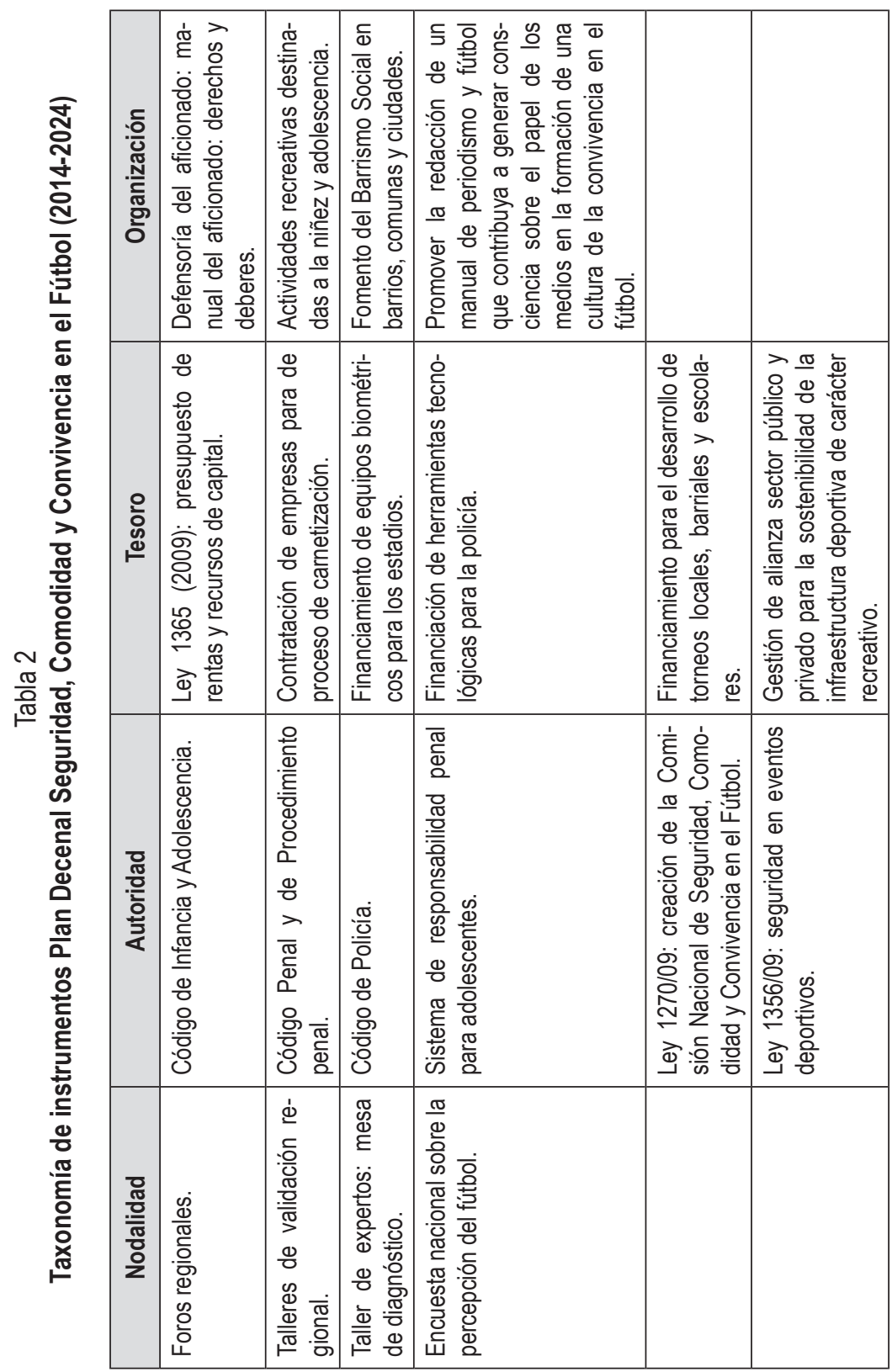




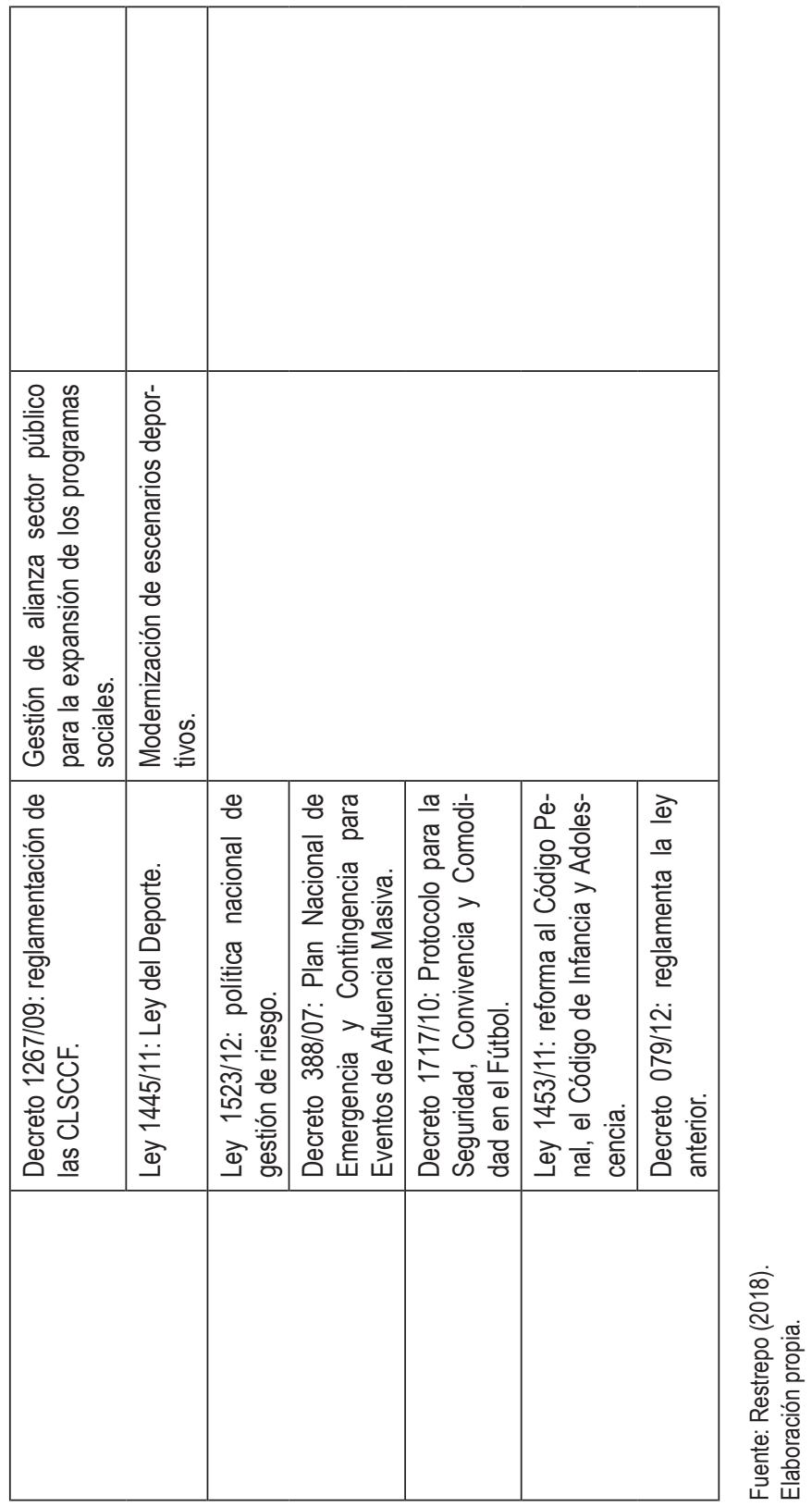


porque simplemente no es rentable invertir en una estructura de carácter público como son los estadios 5 , y mucho menos en unos 'vándalos' que afectan 'el deber ser' de un comportamiento como espectador de fútbol" (Restrepo 2018, 83).

Dentro del enfoque de seguridad, la política se vuelve un negocio. Está el que pone las cámaras, el que pone el biométrico, quien alquila eso, el que te saca el carnet, todo eso. Digamos que, dentro de los intereses del fútbol, teniendo en cuenta que el fútbol es un negocio privado que se juega en escenarios públicos, donde la policía presta la seguridad que es un ente público, o sea, que lo pagamos todos, los intereses del fútbol para que se pueda jugar en una ciudad, el municipio debe tener una inversión amplia para que el estadio tenga las condiciones que el fútbol requiere. El fútbol dentro del negocio privado pues no le interesa un carajo la convivencia, el desarrollo de lo social, ellos quieren el terreno de la seguridad, el sueño del estadio lleno con gente que aplauda sentada, gente que aplauda y no putee, con gente que no diga nada, ¡si pilla! (entrevista a Felipe Garcés 2018).

Con relación a los instrumentos de organización, programas como la Defensoría del Aficionado, a cargo de la Defensoría del Pueblo, "facultada para dar trámite efectivo y seguimiento a las quejas y denuncias presentadas por los aficionados" (CO Ministerio del Interior 2014, 110), no han funcionado debido al alto grado de desconfianza de las barras populares hacia la institucionalidad. El programa creado con el fin de atender las denuncias de los aficionados "existe, pero nadie lo utiliza, las barras a nivel general han optado por una organización en donde los problemas son resueltos internamente por sus integrantes, delegando comisiones de logística, derechos humanos, música, organización de los trapos, etcétera" (Restrepo 2018, 83).

Los casos objeto de este estudio (Medellín y Cali teniendo en cuenta la historia de violencia, criminalidad y desinstitucionalización que poseen y que en la actualidad ha mutado) determinan que es un riesgo denunciar abusos, en especial por parte de la fuerza pública debido a la desconfianza y al temor de una posible represalia. Al respecto, Raúl Martínez, líder de LDS, afirma:

5. Con excepción del Club Deportivo Cali, los demás clubes de fútbol del país juegan en estadios que pertenecen a los distintos municipios en que están afiliados los equipos. 
Si el agredido va poner la denuncia o la demanda lo que pasa en Colombia es que eso entra en un proceso de 10 años donde nunca pasa nada. Tenemos la experiencia de que en Colombia los casos de denuncia frente a agentes oficiales han terminado en el asesinato de los denunciantes ¿qué garantías tenemos nosotros de denunciar a un policía cuando él tiene todos mis datos de donde vivo, de quién es mi familia, todo...? Entonces yo voy y pongo una denuncia contra un policía que me agredió, él me busca en la base de datos interna que tiene el Estado de empadronamiento por el tema del enrolamiento (carnetización), sabe dónde vivo, me manda a matar con los pillos del barrio por la casa de cualquier barrio, aparezco como un asesinado más de la ciudad y ya. (entrevista a Raúl Martínez 2018).

Por otro lado, el factor de la convivencia representado en el fortalecimiento del barrismo social se ha visto limitado por la falta de apoyo del gobierno central hacia los proyectos sociales de las barras, dejando a los municipios la tarea de financiar dichos proyectos. Al respecto Felipe Garcés, líder del BRS comenta:

Estamos hablando de voluntades nacionales que mueven recursos y que deberían a esa política pública iponerle músculo! O sea, proyectos, y como tal meterle recursos a esos proyectos. Entonces, la política es nacional, pero no mete recursos para la ejecución de proyectos, sino que espera que, a nivel local, que es donde se da el fenómeno, se atienda. Que de hecho si uno lo ve eso aparece en la Ley 1270, en donde dice que el Estado a través de sus instituciones garantizará el desarrollo integral de las barras, pero eso no se implementa (entrevista a Felipe Garcés 2018).

A pesar de la escasa inversión de recursos económicos y la poca acogida de los proyectos y programas emprendidos por la política, se puede evidenciar que no solo dichos instrumentos son los que han afectado la implementación del PDSCCF; existe otro factor, y está relacionado con el tipo de interacción entre actores estatales y no estales, es decir, la inclusión de la gobernanza como un paradigma de la administración pública, el cual tiene como objetivo contribuir a la eficiencia del diseño de política públicas por medio de un trabajo mancomunado entre todos los actores involucrados dentro del fenómeno de la violencia asociada al fútbol. 


\section{Procesos de participación en un marco de voluntad política}

La inclusión de diversos actores en los procesos de diseño de políticas públicas como forma de cogobernanza, en términos de Kooiman (2005), ha generado un impacto positivo frente a la manera de acercarse al entendimiento de los fenómenos sociales y, sobre todo, la forma de cómo tratarlos. A pesar del avance de los espacios brindados por la institucionalidad y la activa participación de los movimientos sociales, el diseño de políticas no está exento de estar expuesto a factores como corrupción, voluntad política y su sostenibilidad en el tiempo.

En el campo del diseño de políticas públicas, ciertamente, los intereses de los actores estales no solo direccionan la pertinencia del tratamiento de los fenómenos sociales, sino que además, definen la forma de cómo tratarlos. El concepto de voluntad política evidencia el comportamiento de la administración pública frente a un fenómeno social, en este caso la violencia asociada al fútbol a través de la implementación del PDSCCF. La presión que ejercen los actores no estatales como las barras populares y diversos sectores relacionados con el fútbol profesional, de algún modo, favorece el tratamiento del fenómeno de manera primaria y abre espacios para un trabajo mancomunado; sin embargo, es a final de cuentas la administración pública la que decide cómo trabajar.

El caso de la ciudad de Cali evidencia un esfuerzo por mediar la situación de violencia que se vive producto de las barras de la ciudad. La policía, encabezada por el intendente Rubén Valencia, y las barras populares BRS y FRV han venido trabajando en el desarrollo del barrismo social, dando como resultado programas como la Fiesta por la Vida en los Estadios, "dirigido hacia la vinculación de jóvenes barristas de los estratos socioeconómicos más bajos de la ciudad, en el desarrollo de proyectos productivos, culturales y deportivos" (Garcés 2013, 3). Este programa impulsado desde 2006 contó con el apoyo de la administración Municipal y la Agencia de cooperación internacional OIM, beneficiando a 250 jóvenes de manera directa y 4.500 de forma indirecta (Garcés 2013).

Gracias al impacto del programa, y aprovechando la implementación del PDSCCF, se intentó seguir con su segunda fase denominada Carnaval de 
Difusión Cultural y Caleñidad; sin embargo, este no prosperó debido a la falta de apoyo por parte de la institucionalidad, tal y como lo comenta el intendente de la policía de Cali, Rubén Valencia:

Hemos logrado un avance con lo referido a la Ley. De hecho, fuimos pioneros. Pero luego hubo un abandono sobretodo del programa de parte de la alcaldía, porque se dedicaron a sacar los proyectos, pero no establecieron el programa. Y pues esa es la gran falencia que yo como coordinador del programa de barras en Cali denoto, porque si nosotros tuviéramos un programa avanzaríamos mucho más en el tema social y avanzaríamos mucho más en tener la barra en otro tipo de dinámicas no ligadas tanto a la delincuencia ni a la violencia (entrevista al intendente Rubén Valencia 2018).

Según datos de la alcaldía de Cali, el primer semestre de 2018 se registraron cinco riñas al interior del estadio y veinte en las afueras, se decomisaron 644 armas corto punzantes, se arrestaron 364 personas y se ha negado el acceso a 437, sin mencionar que desde el año 2017 se tiene prohibida la entrada al estadio Pascual Guerrero a hinchadas visitantes. Cabe la pena señalar que la violencia que se sigue presentando no tiene siempre como protagonistas a las barras populares. No obstante, lo que realmente se quiere apuntar es que la violencia es un factor recurrente y que la seguridad como objetivo debe ir de la mano con el componente de convivencia - el cual se ha visto limitado-, desaprovechando el potencial de las barras populares como espejo de la sociedad para la atención de problemas sociales, rezagándolas a un plano de participación ínfima en la que lo máximo de participación a la que tiende es que sea de carácter consultivo.

Al respecto, el alcalde actual de la ciudad de Cali, Maurice Armitage, reafirma el estigma de violencia que cargan las barras populares, desconociendo la complejidad del fenómeno y el trabajo social, cultural y productivo que estás llevan a cabo, tal y como lo menciona en el foro "La violencia en el fútbol no aguanta":

Llego a la Alcaldía, y me encuentro con una situación absurda, de que unas barras hay que volverlas interlocutores de la tranquilidad de la ciudad. Dije yo: " ¡iEsta vaina qué es!?". Yo te acepto que nos entendamos con "Tiro Fijo" (comándate de las FARC) para la paz del país, pero sentarme yo, con unos muchachos que los considero, que son en gran mayoría, porque no todos, en gran mayoría son delincuentes, que están utilizando el fútbol para delinquir y propiciar violencia. Pues yo no me puedo entender con ellos, y no me voy a sentar con ellos (entrevista a Armitage 2017). 
Por su parte, el caso de Medellín ha tomado un rumbo diferente en la forma de tratar un fenómeno social como la violencia en el fútbol. La Alcaldía actual se dio a la tarea de retomar todo lo que se había trabajado en materia de fútbol, se propuso dar trámite a lo que desde las barras de la ciudad se está trabajando y presentó nuevas acciones para enfrentar este fenómeno; con la particularidad que dentro de sus acciones contó con la participación de las barras populares.

Dentro de los programas promovidos por las barras, se destaca el ejemplo de "Con la Pelota en la Cabeza". Desarrollado por LDS, este programa consiste en recoger "expresiones artísticas y creaciones de la ciudad en torno al fútbol y su relación con la literatura (escritos), pintura, dibujo, imagen, fotografía, música (canciones y producción discográfica) y cómics (de ámbito latinoamericano)" (Prieto 2017, 7). Su intención se centra en "rescatar diferentes reflexiones y vivencias a través de textos narrativos e ilustraciones, que dibujan la realidad de la ciudad y la sociedad en relación con el fútbol" (Prieto 2017, 6).

Por otro lado, la LDS también cuentan con proyectos de emprendimiento productivo que tienen, como principal objetivo, ofrecer oportunidades de trabajo a los miembros de la barra, dando como resultado la consolidación de una empresa de producción audiovisual y logística llamada "AN Logística". "Con esta empresa que recoge la experiencia de 8 años de trabajo en logística de partidos del Atlético Nacional dentro del Estadio Atanasio Girardot y otros eventos grandes de la ciudad, se genera empleo para más de 300 integrantes de la barra" (Los Del Sur 2017, 11).

La barra RXN viene trabajando con niños de bajos recursos de los barrios marginales de la ciudad a través del desarrollo de una escuela deportiva. Al respecto, Violo, líder de la barra y director de la escuela deportiva, comenta acerca del proceso del programa:

Tenemos en este momento cerca de 120 niños en la escuelita, en los que ningún niño tiene que pagar nada por estar dentro del club de nosotros, es $100 \%$ un trabajo social. A los niños se les da desde el uniforme hasta la instrucción deportiva, inscripción a los torneos y digamos transporte cada 8 días cuando toca salir del barrio de influencia, que toque ir a otros barrios de la ciudad para participar en los diferentes torneos (entrevista a Violo 2018). 
Con el objetivo de promover la cultura de la no violencia en el fútbol, la alcaldía de Medellín se ha enfocado en apoyar el trabajo social y fomentar programas que ayuden a los barristas a crear un ambiente de tolerancia, destacando ejemplos como: el regreso de los clásicos con las barras representativas de la ciudad, juntar las dos bandas musicales de las barras con la banda sinfónica de la universidad de Antioquia y ofrecer conciertos gratis por diversos puntos de la ciudad previo a vivir un clásico, jugar un partido 24 horas entre los miembros de las barras, la institucionalidad y la sociedad civil conocido como "el clásico más largo de la historia", entre otros. Dichos ejemplos, sumado al trabajo social de las barras, han motivado al Concejo Municipal para que acepte implementar una política pública local denominada "Cultura del Fútbol". Encabezada por el concejal Daniel Carvalho, esta política proyecta sostener en el tiempo el apoyo institucional y la asignación de responsables para su ejecución:

Para el año 2015, cuando a los partidos solo podía entrar una barra, el promedio de asistencia de espectadores al estadio era de 28.000 , registrando un promedio de treinta y cinco personas retenidas por partido, un promedio de veinte y cuatro armas blancas y la incautación de 45 porciones de droga. En el 2016 luego de incluir el tema del barrismo en el plan de desarrollo de la ciudad, se jugaron cuatro clásicos con las dos barras presentes. El promedio de asistencia se elevó a 32.000, el promedio de personas retenidas se redujo a veinte y ocho, las armas blancas a catorce y las porciones de droga a veinte y seis. En 2017 la confianza de la sociedad seguía en aumento, los clásicos continuaron con las dos hinchadas, el promedio de espectadores llegó a los 36.000, el promedio de retenidos seguía a la baja con diecisiete, al igual que las armas blancas con once y las porciones de droga con diecisiete. En lo que va corrido del año 2018 el número de espectadores alcanzó un promedio de 41.856 espectadores, sin mencionar que el número de riñas se ha reducido a cero aumentando la tolerancia entre barristas (entrevista a Mateo 2018).

\section{Conclusiones}

No cabe duda de que el fútbol en la actualidad se ha posicionado como relevante para la sociedad, influyendo en varias de sus capas que van desde lo social, cultural, económico hasta influir en decisiones políticas.

El fútbol, y específicamente la afición por este deporte, refleja en cierta medida las capas subalternas de nuestras sociedades y los problemas que 
de ellas derivan. La escalada de violencia que se vive a causa del fanatismo refleja un componente, por un lado, psicológico al ver las barras como un esquema de relaciones de poder, $y$, por el otro, social, al estar implícitas condiciones de marginalidad a las que se ven sometidos muchos de los miembros que las componen.

Como consecuencia de la relevancia del fútbol para la sociedad, los problemas de violencia y vandalismo que de este deporte se derivan han ocasionado la necesidad de que el Estado intervenga para buscar una solución. En esa medida, desde la aparición de los hooligans en Europa hasta el desarrollo de las denominadas barras bravas en el sur del continente americano, la respuesta de los distintos Estados frente a esta problemática ha contado con un común denominador que se expresa en la coerción.

Si bien el contexto latinoamericano es muy distinto al europeo, las formas de contener el flagelo de la violencia pasan por el orden de la vigilancia, el castigo y la fuerza, mas nunca por un componente educativo o de convivencia. Por dicha razón, el caso colombiano resulta ser un hecho inédito en la búsqueda de explorar opciones distintas a la coerción. Es cierto que el fútbol no va a resolver los problemas estructurales de la sociedad, pero sí se puede estructurar como un instrumento que ayude a mitigarlos.

Conforme dicha lógica, el Plan Decenal de Seguridad, Comodidad y Convivencia en el Fútbol se establece como un esfuerzo por intervenir el flagelo de la violencia al integrar la seguridad con el componente de la convivencia a través de un trabajo pedagógico y de desarrollo social. A pesar del gran esfuerzo que implicó diseñar una política a partir de un componente inclusivo, en cuyo proceso de formulación se retroalimentó de diversos grupos de actores tanto estatales como no estatales, la política evidencia ciertas falencias al implementarse, lo cual ineludiblemente se está alejando del cumplimiento de sus objetivos y por lo tanto a su falla.

A causa de sus inconsistencias, la aplicación del modelo anidado multinivel desarrollado por Howlett logró identificar que los instrumentos relacionados con los recursos (tesoro) están presentando dificultades para el respaldo de ciertos programas que impiden que se desarrolle el componente de convivencia. Los recursos destinados han sido principalmente para fortalecer el componente de la seguridad por medio de la instalación de cámaras, equipos biométricos y carnetización de los hinchas, mientras que el apoyo a 
los programas y proyectos para fortalecer el barrismo social es mínimo; sin mencionar el agravante que toda la carga tributaria ha sido atribuida a las administraciones locales, eximiendo de responsabilidades al gobierno nacional y a la DIMAYOR como entidad privada que organiza el fútbol profesional en el país. De igual manera, instrumentos de organización referido al desarrollo de programas, como es el caso del Defensor del Aficionado, no han generado el impacto pretendido debido a la falta de confianza que existe principalmente entre los barristas y la fuerza pública al pretender denunciar un abuso por parte de esta.

A pesar de la presencia de inconsistencias en algunos de los instrumentos de la política, la comparación de los casos de Cali y Medellín permitió visibilizar elementos propios de la gobernanza; específicamente, la inclusión de los barristas en los procesos de implementación de proyectos y programas dentro del objetivo del PDSCCF de apoyar el desarrollo del barrismo social. Sin embargo, los procesos de participación dentro de la ejecución de la política fueron distintos para ambos casos y en consecuencia la efectividad de la misma también lo fue.

El caso de Medellín, por un lado, manifestó resultados positivos con respeto al objetivo de avanzar hacia una cultura de la no violencia de acuerdo a un tipo de participación interactiva. La alcaldía municipal ha decidido trabajar de manera conjunta con las barras locales con la finalidad de apoyar las actividades sociales, culturales y de emprendimiento que desde las barras se ha venido construyendo y que se ha ido consolidando como un esfuerzo de querer derrumbar el estigma de violencia. En esa misma línea la alcaldía no solo apoya el fortalecimiento del barrismo social, sino que, además, configura propuestas que aporten a lo que se está trabajando y así poder llegar por medio del barrismo a los sectores más vulnerables y marginales de la ciudad. De esta manera la administración municipal ha logrado generar procesos de visibilización de las barras, así como espacios de encuentro entre las mismas para que se reconozcan como iguales, fortaleciendo el tema de la tolerancia entre por lo menos las barras de la ciudad.

Por otro lado, el caso de Cali, si bien cuenta con antecedentes de trabajo en conjunto entre instituciones públicas como la Alcaldía y la policía con las barras de la ciudad, la experiencia actual arroja que el máximo nivel de trabajo articulado entre dichos actores se queda en el plano de lo consultivo. 
La implementación del PDSCCF en Cali ha sido infructuosa, la administración local no manifiesta un interés real por apoyar los procesos de barrismo social que se manifiestan en la ciudad, y solo se ha empeñado en dar trámite a la política en cuanto a la necesidad de brindar seguridad al espectáculo deportivo por medio de la instalación de equipos de control de asistencia y de vigilancia.

Por último, vale la pena señalar que los casos estudiados reflejan la importancia de los procesos de inclusión de actores no estatales (en este caso de las barras) dentro del diseño de políticas. Sin embargo, los casos referidos en este estudio también arrojaron elementos subjetivos que influyen en la efectividad del diseño de políticas, así como en las formas de participación, tal y como es la voluntad política. Dicha voluntad, de cierta manera, dentro del debate de la gobernanza en el caso de Medellín, permitió avanzar en el objetivo de mitigar la violencia y aprovechar el potencial de la cultura que envuelve al fútbol como una oportunidad para la reconstrucción del tejido social; mientras que, en el caso de Cali, es evidente la falta de interés por parte de su administración de querer apoyar formas alternativas a la coerción como el barrismo social que permitan mitigar la violencia en el fútbol y los riegos que de ella germinan.

\section{Referencias}

Aguilar Villanueva, Luis. 2006. Gobernanza y gestión pública. Ciudad de México: Fondo de Cultura Económica.

Armitage, Maurice. 2017. "Panel 2: seguridad y convivencia". Video de YouTube emitido por la Procuraduría General de la Nación. Consulta: noviembre de 2018. «https://www. youtube.com/watch? $\mathrm{v}=8 \mathrm{hlFtDE} 3 J \mathrm{JD} 0 \& \mathrm{t}=719 \mathrm{~s}$ >

Cerrillo, Agustín. 2005. "La gobernanza hoy: introducción". En Agustín Cerrillo, coord., La gobernanza hoy: 10 textos de referencia, 11-35. Madrid: Instituto Nacional de Administración Pública.

CO Ministerio del Interior. 2014. "Plan Decenal de Seguridad, Comodidad y Convivencia en el fútbol (2014-2024)". Bogotá: Editorial Gente Nueva.

Córdova, Marco. 2018. "La incidencia de la gobernanza en el diseño de las políticas de reconstrucción postdesastres. Análisis comparado en América Latina". Documento de trabajo. Quito: FLACSO Ecuador. 
Critchley, Simon. 2018. En qué pensamos cuando pensamos en fútbol. Editado por Milo Krmpotic. Madrid: Sexto Piso.

Domínguez, Juan Camilo. 2009. "Con Los Del Sur en la cabeza”. Ponencia presentada en el XXVII Congreso de la Asociación Latinoamericana de Sociología. Buenos Aires, 15 de noviembre.

Garcés, Felipe. 2013. "Experiencias culturales de las barras populares". Documento de trabajo. Bogotá: Ministerio de Cultura Colombia.

Howlett, Michael. 2009. "Governance Modes, Policy Regimes and Operational Plans: A Multi-Level Nested Model of Policy Instrument Choice and Policy Design". Policy Sciences 42, No. 1: 73-89.

Kooiman, Jan. 2005. "Gobernar en gobernanza”. En Agustín Cerrillo, coord., La gobernanza hoy: 10 textos de referencia, 57-82. Madrid: Instituto Nacional de Administración Pública.

Los Del Sur. 2017. "Los Del Sur, 20 años de trabajo social y popular. Nuestro aporte a la convivencia”. En Los Del Sur, edit., Con la pelota en la cabeza, 10-12. Medellín: Los Del Sur.

McConnell, Allan. 2015. "¿What is Policy Failure? A Primer to Help Navigate the Maze”. Public Policy and Administration 30, No. 3: 221-242.

Murad, Mauricio. 2013. "Violências e mortes no futebol brasileiro: Reflexões, investigações, proposições". Revista Portuguesa de Ciencias del Deporte 13, No. 1: 57-72.

Pardey, Harold, Juan Galeano y Andrés Blanco. 2001. La Ciudad de los fanáticos: aproximación al fenómeno de las barras de fútbol locales Barón Rojo Sur y Frente Radical Verdiblanco, entre los años 1999 y 2001. Cali: Universidad del Valle.

Prieto, Eugenio. 2017. "Prólogo". En Los Del Sur, edit., Con la pelota en la cabeza, 6-7. Medellín: Los Del Sur.

Przeworski, Adam, y Henry Teune. 1982. The Logic of Comparative Social Inquiry. Malabar (FL): Krieger Publishing.

Restrepo, Juan Manuel. 2018. "Plan decenal de seguridad, comodidad y convivencia en el fútbol: entre la vigilancia y la voluntad política, un análisis comparado sobre la política del fútbol colombiano los casos de Cali y Medellín”. Tesis de maestría en Estudios Urbanos. Facultad Latinoamericana de Ciencias Sociales, Quito (FLACSO Ecuador).

Rojas, Lina. 2013. "Las barras bravas como una tribu urbana: una búsqueda de identidad. Estudio de caso del Barón Rojo Sur y Frente Radical Verde". Tesis de pregrado. Universidad del Valle, Cali.

Sánchez, Juan. 2012. "Usos de los conceptos de gobernabilidad y gobernanza (una manera de diferenciarlos)". En Bertha Lerner, Ricardo Uvalle y Roberto Moreno, coords., Gobernabilidad y gobernanza en los albores del siglo XXI y reflexiones sobre el México contemporáneo, 217-265. Ciudad de México: Universidad Nacional Autónoma de México-Instituto de Investigaciones Sociales.

Santillán Mileny. 2004. "Criterios metodológicos para construir tipologías de sistemas de gestión participativa municipal". Tesis de maestría en Desarrollo Local. FLACSO Ecuador. 
Consulta: octubre de 2017. «http://repositorio.flacsoandes.edu.ec/bitstream/10469/652/8/ TFLACSO-04-2004MSN.pdf〉.

\section{Entrevistas (2018)}

Garcés, Felipe. Líder barrista (BRS).

Martínez, Raúl. Líder barrista (LDS).

Mateo. Funcionario de la Secretaría de seguridad y convivencia a cargo del tema de barras (Alcaldía de Medellín).

Valencia, Rubén. Intendente de la Policía Nacional.

Violo. Líder barrista (RXN). 